\title{
UNIVERSAL ABELIAN COVERS OF QUOTIENT-CUSPS
}

\author{
WALTER D. NEUMANN AND JONATHAN WAHL
}

\begin{abstract}
The quotient-cusp singularities are isolated complex surface singularities that are double-covered by cusp singularities. We show that the universal abelian cover of such a singularity, branched only at the singular point, is a complete intersection cusp singularity of embedding dimension 4 . This supports a general conjecture that we make about the universal abelian cover of a $\mathbb{Q}$-Gorenstein singularity.
\end{abstract}

\section{INTRODUCTION}

In this paper we introduce a general conjecture, and prove it in a new case. Recall that a normal surface singularity is called $\mathbb{Q}$-Gorenstein if the dualizing sheaf $\omega_{X}$ has finite order. Equivalently, for some $n>0, \Gamma\left(X-\{o\}, \omega_{X}^{\otimes n}\right)$ is a free $\mathcal{O}_{X}$-module; or, $(X, o)$ is the quotient of a Gorenstein singularity by a finite cyclic group acting freely off the singular point.

Conjecture 1.1. Let $(X, o)$ be a $\mathbb{Q}$-Gorenstein normal surface singularity whose link is a rational homology sphere. Then the universal abelian cover $(\tilde{X}, o)$ of $(X, o)$ is a complete intersection, of embedding dimension at most the number of ends in the resolution graph of $(X, o)$.

Our notation $(X, o)$ implies that we are considering the germ of a normal complex surface singularity. Any finite connected covering of the link of this singularity gives rise naturally to a unique analytic covering of $(X, o)$ branched only at the singular point (namely, form the cover $Z \rightarrow X-\{o\}$ and then take $\operatorname{Spec} \Gamma\left(\mathcal{O}_{Z}\right)$ ). If the link is a rational homology sphere, then its universal abelian covering is a finite covering, so it gives rise to the so-called universal abelian covering of the corresponding singularity.

We shall discuss special cases of Conjecture 1.1 that suggest that the complete intersection in the conjecture may be of quite special type. But first we discuss some of the background of this conjecture.

For many years, the second author has been mystified by the appropriate philosophical context by which to understand a theorem of the other author, namely

Theorem $1.2([8])$. Let $(X, o)$ be a weighted homogeneous normal surface singularity whose link is a rational homology sphere. Then the conclusion of Conjecture 1.1 applies. In fact, the universal abelian cover $(\tilde{X}, o)$ of $(X, o)$ is a Brieskorn complete intersection.

2000 Mathematics Subject Classification. 14B05, 14J17, 32S25.

Key words and phrases. cusp singularity, Gorenstein surface singularity, complete intersection singularity.

This research was supported by grants from the Australian Research Council and the NSF (first author) and the the NSA (second author). 
The proof of the above theorem is completely convincing as well as totally unenlightening: given the data determining the analytic type of $(X, o)$, one writes down explicit equations of a Brieskorn complete intersection plus an explicit action of an abelian group $G$, and then argues directly that $G$ acts freely off the origin $o$, with quotient isomorphic to $(X, o)$, and that $G$ has the correct order.

It is easy to see that $(X, o)$ is $\mathbb{Q}$-Gorenstein in the above theorem, and that $(\tilde{X}, o)$ must therefore be Gorenstein. But, there is no useful general result limiting the topology of complete intersection singularities among the Gorenstein ones. So the statement that a topologically defined Gorenstein singularity is a complete intersection must be surprising (as also the very special form: Brieskorn complete intersection). There is one simple case for the theorem: the universal abelian cover of a quotient singularity $\mathbb{C}^{2} / G$ (with $G \subset \mathrm{GL}(2, \mathbb{C})$ finite, acting freely off $\left.(0,0)\right)$ is clearly $\mathbb{C}^{2} / G^{\prime}$, where $G^{\prime}$ is the commutator subgroup. But then $G^{\prime} \subset \operatorname{SL}(2, \mathbb{C})$, so $\mathbb{C}^{2} / G^{\prime}$ must be smooth or a rational double point, and necessarily a hypersurface. In fact it is of type $A_{n}, D_{4}, E_{6}$, or $E_{8}$, the only rational double points which are Brieskorn singularities.

The next impetus towards Conjecture 1.1 was our study of normal surface singularities whose links are integral homology spheres, developing out of [9]. A $\mathbb{Q}$ Gorenstein singularity of this type is necessarily Gorenstein, but the conjecture would imply that it is always a complete intersection! This issue was discussed by us in [9], and will be examined more closely in a forthcoming paper [10]. There are a number of reasons to believe the conjecture in this case, and this would imply completely unexpected topological restrictions on links of such singularities.

Conjecture 1.1 applies to rational surface singularities, but even the weaker result that every rational surface singularity is the quotient of some complete intersection singularity is unknown.

Note finally that an equisingular deformation of a singularity satisfying the Conjecture (e.g., a deformation of a weighted homogeneous singularity as in Theorem 1.2) does not itself obviously satisfy the conjecture! One problem is that if $(Y, o) \rightarrow(X, o)$ is a finite covering (unramified off $o$ ), an equisingular deformation of $X$ gives rise to a deformation of $Y-\{o\}$, but not a priori to one of $Y$.

From the point of view of classification of singularities, the easiest examples not covered by Theorem 1.2 are the quotient-cusps. These are singularities whose resolution graphs have the form

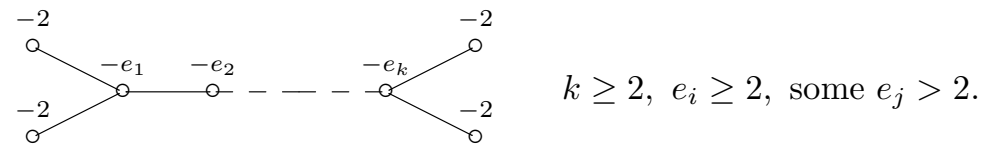

They are rational singularities, even log-canonical. Like the cusps, they are taut, i.e, the topology of the link determines the analytic type of the singularity [5]. (See, e.g., [4] for a discussion of log-canonicals, and [1], [5], [3], [1] and [7] for discussions of cusps and quotient-cusps. The only log-canonical singularities which are not weighted homogeneous are the cusps and quotient-cusps.)

The above quotient-cusp is double-covered by the cusp singularity whose resolution graph is

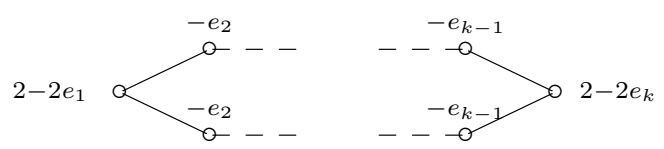


It follows that the universal abelian cover is also a cusp. While it is easy to determine, given the resolution graph, when a cusp is a complete intersection (Proposition 2.5 below), there is still significant work to prove the surprising (but consistent): The universal abelian cover of a quotient-cusp is a complete intersection of embedding dimension 4.

To describe the precise result we need some notation. We associate to the quotient-cusp singularity described above the matrix

$$
B=\left(\begin{array}{ll}
a & b \\
c & d
\end{array}\right)=B\left(e_{1}-1, e_{2}, \ldots, e_{k-1}, e_{k}-1\right)
$$

with

$$
B\left(b_{1}, \ldots, b_{k}\right):=\left(\begin{array}{cc}
0 & 1 \\
-1 & 0
\end{array}\right)\left(\begin{array}{cc}
0 & -1 \\
1 & b_{k}
\end{array}\right) \ldots\left(\begin{array}{cc}
0 & -1 \\
1 & b_{1}
\end{array}\right) .
$$

We shall see below (Lemma 2.1) that $B$ is an integer unimodular matrix with positive entries, and each unimodular integer matrix with positive entries arises this way for unique $e_{1}, \ldots, e_{k}$ as above. Reversing the order of $e_{1}$ to $e_{k}$ just exchanges the entries $a$ and $d$. Thus isomorphism types of quotient-cusp singularities are in one-one correspondence with unimodular $2 \times 2$ matrices $\left(\begin{array}{ll}a & b \\ c & d\end{array}\right)$ with positive integer entries $a, b, c, d$, up to exchanging $a$ and $d$.

Theorem 1.3. If $(V, p)$ is the quotient-cusp classified as above by $\left(\begin{array}{ll}a & b \\ c & d\end{array}\right)$, then its universal abelian cover branched at $p$ is the complete intersection cusp singularity given by equations

$$
x y=u^{2 a}+v^{2 a} ; \quad u v=x^{2 d}+y^{2 d}
$$

The covering degree is $16 b$.

The cusp singularity in question is the one with resolution graph

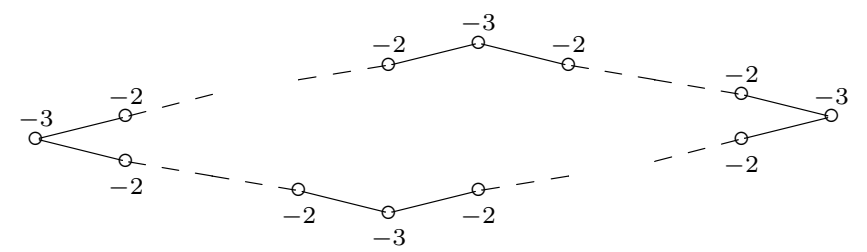

where the four strings of -2 's are lengths $2 a-3,2 d-3,2 a-3$, and $2 d-3$. If $d=1$ or $a=1$ this must be replaced by

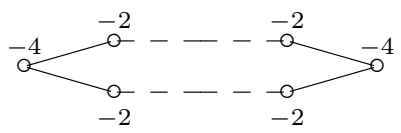

where the top and bottom strings are length $2 a-3$ or $2 d-3$. The above equations for these cusps were given by Karras [3].

The point of our proof is that a cusp or quotient-cusp is determined by its topology, so the proof becomes a purely topological (in fact group-theoretical) calculation.

A quotient-cusp turns out to have other abelian covers which are complete intersections, but we have no "reason" for it, except calculation. Also, any cusp singularity has a finite cover by a complete intersection, but there are cusps with no abelian cover by a complete intersection.

In the final section we sketch a more geometric proof of the theorem by giving an explicit diagonal group action on a (necessarily different) complete intersection 
representation of our cusp. This is in the spirit of the proof in [8] of Theorem 1.2 , and borrows from themes we develop further in [10]. But the verification that the quotient of the action is correct is more difficult than in [8]. So, as presented here, this proof still depends on our earlier computations, and for simplicity we only give full details for $b$ odd.

\section{REVIEW OF CUSPS AND QUOTIENT-CUSPS}

In this section we review basic facts about cusp and quotient-cusp singularities. Before describing the topological significance of the classifying matrix for the quotient-cusp we prove the properties mentioned above. The above matrix

$$
B\left(b_{1}, \ldots, b_{k}\right):=\left(\begin{array}{cc}
0 & 1 \\
-1 & 0
\end{array}\right)\left(\begin{array}{cc}
0 & -1 \\
1 & b_{k}
\end{array}\right) \ldots\left(\begin{array}{cc}
0 & -1 \\
1 & b_{1}
\end{array}\right) .
$$

is clearly a unimodular integer matrix.

Lemma 2.1. If $e_{i} \geq 2$ for each $i=1, \ldots, k$ and at least one $e_{i} \geq 3$, then

$$
B\left(e_{1}-1, e_{2}, \ldots, e_{k-1}, e_{k}-1\right)
$$

has positive entries. Conversely, any unimodular integer matrix with positive entries has a unique representation in the above form.

Proof. Consider a product of matrices

$$
\left(\begin{array}{cc}
-\gamma & -\delta \\
\alpha & \beta
\end{array}\right)=\left(\begin{array}{cc}
0 & -1 \\
1 & b_{k}
\end{array}\right) \ldots\left(\begin{array}{cc}
0 & -1 \\
1 & b_{1}
\end{array}\right)
$$

with $k>1$. The first statement of the lemma can be reformulated that if $b_{1} \geq 1$, $b_{i} \geq 2$ for $1<i \leq k$ and $b_{k} \geq 1$, with at least one of these inequalities strict, then $\alpha, \beta, \gamma, \delta$ are all positive.

We will show, in fact, that $\alpha, \beta, \gamma, \delta$ are all positive with the largest given by the following table:

$$
\begin{aligned}
\beta \text { largest } & \Longleftrightarrow b_{1}>1 \text { and } b_{k}>1 \\
\alpha \text { largest } & \Longleftrightarrow b_{1}=1<b_{k} \\
\delta \text { largest } & \Longleftrightarrow b_{1}>1=b_{k} \\
\gamma \text { largest } & \Longleftrightarrow b_{1}=1=b_{k}
\end{aligned}
$$

The cases $\left(b_{1}, \ldots, b_{k}\right)=(1, b),(b, 1)$, or $(1, b, 1)$ can be quickly verified by hand; we leave this to the reader.

Next suppose $b_{1} \geq 2$ and $b_{k} \geq 2$. A simple induction on $k$ shows that the entries of the product then satisfy $0<\gamma, \gamma<\alpha<\beta$, and $\gamma<\delta<\beta$. This proves the first case of the above table. Moreover, the same induction shows that $\beta+\gamma-\alpha-\delta>0$ unless $b_{1}=\cdots=b_{k}=2$. By post- and/or pre-multiplying by $\left(\begin{array}{cc}0 & -1 \\ 1 & 1\end{array}\right)$ one now easily completes the proofs of the other three cases of the table.

For the converse, consider $\left(\begin{array}{cc}-\gamma & -\delta \\ \alpha & \beta\end{array}\right)$ with $\alpha, \beta, \gamma, \delta>0$ and $\alpha \delta-\beta \gamma=1$. Assume first that $\beta$ is the largest of $\alpha, \beta, \gamma, \delta$. Then we must have $\gamma<\alpha<\beta$ and $\gamma<\delta<\beta$. Put $b_{1}=\left\lceil\frac{\delta}{\gamma}\right\rceil$. If we write

$$
\left(\begin{array}{cc}
-\gamma & -\delta \\
\alpha & \beta
\end{array}\right)=\left(\begin{array}{cc}
-\gamma^{\prime} & -\delta^{\prime} \\
\alpha^{\prime} & \beta^{\prime}
\end{array}\right)\left(\begin{array}{cc}
0 & -1 \\
1 & b_{1}
\end{array}\right)
$$

then $\alpha^{\prime}=b_{1} \alpha-\beta, \beta^{\prime}=\alpha, \gamma^{\prime}=b_{1} \gamma-\delta$ and $\delta^{\prime}=\gamma$. Thus, if $\gamma \neq 1$ we have $\gamma^{\prime}>0$. Also, $\alpha^{\prime}>\frac{\delta}{\gamma} \alpha-\beta=\frac{1}{\gamma}>0$, and $\delta^{\prime}<\beta^{\prime}$ and $\alpha^{\prime}=b_{1} \alpha-\beta<\left(1+\frac{\delta}{\gamma}-\frac{1}{\alpha \gamma}\right) \alpha-\beta=\beta^{\prime}$. 
It follows that $\alpha^{\prime}, \beta^{\prime}, \gamma^{\prime}, \delta^{\prime}$ are all positive and $\beta^{\prime}$ is the largest. Thus we can proceed inductively. The value of $\gamma$ is easily seen to decrease at each inductive step, and when $\gamma=1$ we have $\gamma^{\prime}=0$, so we can write

$$
\left(\begin{array}{cc}
-\gamma^{\prime} & -\delta^{\prime} \\
\alpha^{\prime} & \beta^{\prime}
\end{array}\right)=\left(\begin{array}{cc}
0 & -1 \\
1 & b_{k}
\end{array}\right)
$$

Thus, our matrix $\left(\begin{array}{cc}-\gamma & -\delta \\ \alpha & \beta\end{array}\right)$ is a product of the desired type with all $b_{i} \geq 2$.

The other three cases of the above table now follow easily as before by first postand/or pre-multiplying $\left(\begin{array}{cc}-\gamma & -\delta \\ \alpha & \beta\end{array}\right)$ by the inverse of $\left(\begin{array}{cc}0 & -1 \\ 1 & 1\end{array}\right)$.

Finally, the uniqueness of the representation follows by noting that the value we chose for $b_{1}$ in the above argument is forced by the fact that $\gamma^{\prime} \geq 0$ by the calculation of the first part of the proof.

Let $M$ denote the oriented 3-manifold that is the total space of the unit tangent bundle of the Möbius band. This bundle has a section consisting of unit tangent vectors that are parallel to the core circle of the Möbius band (once this circle is oriented); this also provides a section on the boundary $\partial M$. Thus we have a natural identification $\partial M \cong S^{1} \times S^{1}$ where the first $S^{1}$ is base and the second $S^{1}$ is fiber.

Lemma 2.2. The link $N$ of the quotient-cusp with resolution graph

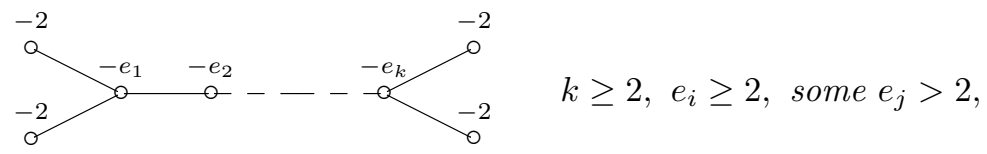

is the 3-manifold obtained as $N=M \cup_{\phi} M$, where $\phi$ is the pasting map $\phi: \partial M=$ $T^{2} \rightarrow T^{2}=\partial M$ given by the matrix $B=B\left(e_{1}-1, e_{2}, \ldots, e_{k-1}, e_{k}-1\right)$ and $N$ is oriented with the orientation of the first $M$.

Conversely, if the link of a singularity is diffeomorphic to $M \cup_{\phi} M$ for some $\phi: T^{2} \rightarrow T^{2}$ given by a unimodular matrix with positive entries, then the singularity is the corresponding quotient-cusp. In fact, it suffices that the link have the correct fundamental group.

Since $\phi$ is orientation preserving in this lemma, $N$ inherits opposite orientations from its two pieces $M$, which is why we must specify that we take the orientation inherited from the first.

Proof. As described in [7] (see "Step 3" on p. 314), $N$ is obtained by plumbing from the plumbing diagram

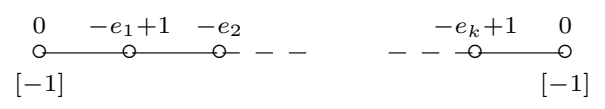

The two end vertices of this plumbing diagram give the pieces $M$ that are pasted. As computed on page 319 of [7], the pasting map is given by the matrix product $J H_{k} J \ldots J H_{1} J$, where $J=\left(\begin{array}{rr}0 & 1 \\ 1 & 0\end{array}\right)$ and $H_{i}=\left(\begin{array}{cc}-1 & 0 \\ b_{i} & 1\end{array}\right)$ with $b_{i}=e_{i}$ or $e_{i}-1$ according as $1<i<k$ or $i=1, k$. This gives the claimed gluing matrix after left-multiplying by $\left(\begin{array}{cc}1 & 0 \\ 0 & -1\end{array}\right)$ to make the gluing map unimodular (this reverses the orientation of the second $M$ ). 
The final part of the lemma says quotient-cusps are taut, in the sense that a singularity with the same topology as a quotient-cusp is analytically that quotientcusp. This was proved by Laufer [5]. The fact that the fundamental group suffices to determine the topology is part of Corollary 4 of [7].

A cusp singularity is one whose minimal resolution graph is a cycle of vertices representing rational curves $E_{i}$. We shall denote this graph by $\left[-e_{1}, \ldots,-e_{k}\right]$ (this notation is well-defined up to cyclic permutation and reversal), where $-e_{i}$ is the Euler number of the normal bundle of $E_{i}$. If $k>1$ then $-e_{i}$ is the self-intersection number $E_{i} \cdot E_{i}$; but if $k=1$ then $E_{1} \cdot E_{1}=-e_{1}+2$, since $E_{1}$ intersects itself normally in one point. The $e_{i}$ satisfy $e_{i} \geq 2$ for all $i$ and some $e_{j} \geq 3$.

Lemma 2.3. The cusp singularity with resolution graph $\left[-e_{1}, \ldots,-e_{k}\right]$ has link the torus bundle over $S^{1}$ with monodromy

$$
A=\left(\begin{array}{cc}
0 & -1 \\
1 & e_{k}
\end{array}\right) \ldots\left(\begin{array}{cc}
0 & -1 \\
1 & e_{1}
\end{array}\right) .
$$

This matrix $A$ has trace $\geq 3$. The fundamental group of this singularity link is the semi-direct product $\mathbb{Z}^{2} \rtimes \mathbb{Z}$ where a generator of $\mathbb{Z}$ acts on $\mathbb{Z}^{2}$ by the matrix $A$.

Conversely, every torus bundle over $S^{1}$ with monodromy matrix $A$ having trace $\geq 3$ is the link of a singularity: such a matrix is conjugate to a matrix as above and the singularity is the corresponding cusp.

Proof. See [7]. The second part of the last sentence is again Laufer's tautness of cusp singularities [5]. Our orientation convention here is different from [7] (where it was not made explicit), so the matrix given there was $J A J$ rather than $A$.

Although we will not need it, it is worth remarking that the cusp double cover of the quotient-cusp with classifying matrix $\left(\begin{array}{ll}a & b \\ c & d\end{array}\right)$ is the cusp with monodromy $\operatorname{matrix}\left(\begin{array}{ll}d & b \\ c & a\end{array}\right)\left(\begin{array}{ll}a & b \\ c & d\end{array}\right)$.

If one reverses the orientation of a torus bundle over the circle with monodromy $A$ one gets a torus bundle with monodromy $A^{-1}$. Since this does not change the trace of $A$, Lemma 2.3 implies that if one starts with the link of a cusp singularity then the result is the link of another cusp singularity. This is called the dual cusp. The following is well-known (e.g., [1], [7]).

Lemma 2.4. To find the resolution graph of the dual cusp for a given cusp, replace each vertex weight $-e$ with $e \geq 3$ in the resolution graph by a string of $e-3$ vertices weighted -2 , and replace each intervening string of $d$ vertices weighted $-2(d \geq 0)$ by a single vertex weighted $-d-3$.

In particular, this lemma implies that the dual cusp to the cusp with resolution cycle $\left[-e_{1}, \ldots,-e_{k}\right]$ has resolution cycle of length

$$
\sum_{i=1}^{n}\left(e_{i}-2\right) \text {. }
$$

For example, the resolution cycle for the dual cusp to the cusp of Theorem 1.3 is $[-2 a,-2 d,-2 a,-2 d]$ of length 4 .

This is useful in understanding which cusps are complete intersections. U. Karras proved: 
Proposition 2.5 ([2]). The cusp with resolution graph $\left[-e_{1}, \ldots,-e_{n}\right]$ is a complete intersection if and only if $\sum_{i=1}^{n}\left(e_{i}-2\right) \leq 4$. That is, the dual cusp has resolution cycle of length at most 4 .

\section{Proof of Theorem 1.3}

Let $N$ and $M$ be as in Lemma 2.2.

$\pi_{1}(M)=\left\langle x, y \mid x^{-1} y x=y^{-1}\right\rangle$, with $x$ representing a core circle of the Möbius band and $y$ representing the fiber. The subgroup $\pi_{1}(\partial M)$ is generated by $x^{2}$ and $y$. The van-Kampen theorem thus gives a presentation

$$
\begin{aligned}
\pi_{1}(N)=\left\langle x_{1}, y_{1}, x_{2}, y_{2}\right| x_{1}^{-1} y_{1} x_{1} & =y_{1}^{-1}, \quad x_{2}^{-1} y_{2} x_{2}=y_{2}^{-1}, \\
x_{2}^{2} & \left.=x_{1}^{2 a} y_{1}^{c}, \quad y_{2}=x_{1}^{2 b} y_{1}^{d}\right\rangle
\end{aligned}
$$

Abelianizing this presentation shows

$$
\begin{aligned}
\pi_{1}(N)^{a b}=\left\langle X_{1}, Y_{1}, X_{2}, Y_{2}\right| & 2 Y_{1}=0, \quad 2 Y_{2}=0, \\
2 X_{2} & \left.=2 a X_{1}+c Y_{1}, \quad Y_{2}=2 b X_{1}+d Y_{1}\right\rangle
\end{aligned}
$$

The relation matrix

$$
\left(\begin{array}{cccc}
0 & 2 & 0 & 0 \\
0 & 0 & 0 & 2 \\
2 a & c & -2 & 0 \\
2 b & d & 0 & -1
\end{array}\right)
$$

of this abelian presentation has determinant $-16 b$, so the order of $\pi_{1}(N)^{a b}$ is $16 b$.

The fundamental group of the universal abelian cover $\tilde{N}$ of $N$ is

$$
H:=\operatorname{Ker}\left(\pi_{1}(N) \rightarrow \pi_{1}(N)^{a b}\right) .
$$

We claim

\section{Lemma 3.1.}

$$
H=\left\langle s, x_{1}^{4 b}, y_{1}^{2}\right\rangle=\left\langle s, x_{2}^{4 b}, y_{2}^{2}\right\rangle
$$

where

$$
s=\left(x_{1} x_{2}\right)^{2} x_{1}^{-2 a-2} y_{1}^{-c} .
$$

Proof. Denote by $N_{1}$ the link of the cusp singularity that canonically double-covers our quotient-cusp. Since this is an abelian cover, $N_{1}$ is covered by the universal abelian cover $\tilde{N}$ of $N$.

$N_{1}$ fibers over the circle $S^{1}$ with fiber the torus $T^{2}$, giving a semi-direct product representation

$$
\pi_{1}\left(N_{1}\right)=V \rtimes C,
$$

where $V=\pi_{1}\left(T^{2}\right)$ is free abelian of rank 2 and $C=\pi_{1}\left(S^{1}\right)$ is infinite cyclic.

$V$ is the abelian subgroup of $\pi_{1}(N)$ generated by $x_{1}^{2}$ and $y_{1}$. The elements $x_{2}^{2}$ and $y_{2}$ are related to these by the unimodular relations

$$
\begin{aligned}
& x_{2}^{2}=x_{1}^{2 a} y_{1}^{c} \\
& y_{2}=x_{1}^{2 b} y_{1}^{d}
\end{aligned} \quad \text { with inverse } \quad \begin{aligned}
& x_{1}^{2}=x_{2}^{2 d} y_{2}^{-c} \\
& y_{1}=x_{2}^{-2 b} y_{2}^{a} .
\end{aligned}
$$

Thus $x_{2}^{2}$ and $y_{2}$ also generate $V$. We can take $C$ to be generated by the element

$$
z=x_{1} x_{2},
$$

so the subgroup of $\pi_{1}(N)$ generated by $z$ and $V$ is normal of index 2 .

Now the subgroup $V_{1}$ of $V$ generated by $x_{1}^{4 b}$ and $y_{1}^{2}$ clearly has index $4 b$ in $V$. Since $s$ equals $z^{2}$ modulo $V$, the subgroup generated by $s$ and $V_{1}$ has index $8 b$ 
in $\pi_{1}\left(N_{1}\right)$, hence index $16 b$ in $\pi_{1}(N)$. On the other hand, it is evident from the presentation of $\pi_{1}(N)^{a b}$ that $s, x_{1}^{4 b}$, and $y_{1}^{2}$ are in $H$. It follows that the subgroup they generate is $H$ as claimed.

Finally, the above relations show that $V_{1}$ is also generated by $x_{2}^{4 b}$ and $y_{2}^{2}$, giving the second description of $H$ in the lemma.

The above proof gives a semi-direct product representation

$$
H=V_{1} \rtimes S
$$

where $S$ is infinite cyclic, generated by $s$. The conjugation action of $s$ on $V_{1}$ is the same as the action of $z^{2}$, so we first compute the action of $z$ on $V_{1}$. This will tell us the covering $\hat{N}$ of $N_{1}$ determined by the subgroup $\left\langle V_{1}, z\right\rangle$ of $\pi_{1}\left(N_{1}\right)$, and $\tilde{N}$ is then isomorphic to a double covering of $\hat{N}$ ( $\hat{N}$ is not necessarily itself a covering of $N)$. We have

$$
\begin{gathered}
z^{-1} x_{1}^{4 b} z=x_{2}^{-1} x_{1}^{-1} x_{1}^{4 b} x_{1} x_{2}=x_{2}^{-1} x_{2}^{4 b d} y_{2}^{-2 b c} x_{2}=x_{2}^{4 b d} y_{2}^{2 b c}= \\
\left(x_{1}^{2 a} y_{1}^{c}\right)^{2 b d}\left(x_{1}^{2 b} y_{1}^{d}\right)^{2 b c}=\left(x_{1}^{4 b}\right)^{a d+b c}\left(y_{1}^{2}\right)^{2 b c d} \\
z^{-1} y_{1}^{2} z=x_{2}^{-1} y_{1}^{-2} x_{2}=x_{2}^{-1} x_{2}^{4 b} y_{2}^{-2 a} x_{2}=x_{2}^{4 b} y_{2}^{2 a}= \\
\left(x_{1}^{2 a} y_{1}^{z}\right)^{2 b}\left(x_{1}^{2 b} y_{1}^{d}\right)^{2 a}=\left(x_{1}^{4 b}\right)^{2 a}\left(y_{1}^{2}\right)^{a d+b c}
\end{gathered}
$$

Thus $z$ acts on $V_{1}$ by the matrix

$$
\left(\begin{array}{cc}
a d+b c & 2 a \\
2 b c d & a d+b c
\end{array}\right)
$$

This matrix is conjugate by $\left(\begin{array}{cc}0 & -1 \\ 1 & d\end{array}\right)$ to the inverse of

$$
\left(\begin{array}{cc}
0 & -1 \\
1 & 2 a
\end{array}\right)\left(\begin{array}{cc}
0 & -1 \\
1 & 2 d
\end{array}\right)
$$

So $\hat{N}$ is the orientation reversal of the link of the cusp with resolution graph

$$
-2 a \propto-2 d \text {. }
$$

That is, $\hat{N}$ is the link of the dual cusp to this cusp. By Lemma 2.4 the dual cusp in this case has graph $(a, d>1)$

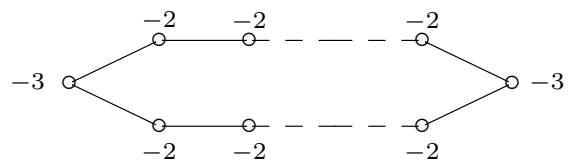

with the top and bottom strings of -2 's of length $2 a-3$ and $2 d-3$. If $a$ or $d$ is 1 the graph is

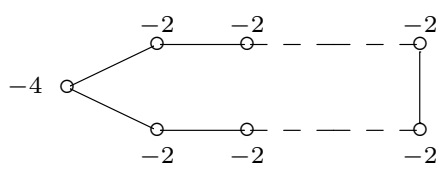

with $2 d-3$ or $2 a-3$ vertices weighted -2 .

Now the cusp we really want is the double cover whose monodromy acts as the square of $z$ rather than $z$. It therefore has resolution graph the double cover of the above cycle. This is as claimed after Theorem 1.3, and the fact that this is a complete intersection with the equations as described there can be found in [3]. This completes the proof. 
Although we did not discuss orientations in detail, we were careful to choose fundamental group generators appropriate to orientation in the above proof. This is important: the other orientation of our link would mean we have the dual cusp, which is a complete intersection only if $a+d=3$. To be sure that orientations are correct we will check a simple case by another method.

We first introduce terminology. A link of a cusp singularity has a fibration $N \rightarrow S^{1}$ with torus fibers. A covering of $N$ that pulls back from a cyclic covering of the base $S^{1}$ will be called a covering in the circle direction. At the opposite extreme, a fiberwise covering will be one that induces the identity map on the base circles of the torus fibrations.

Not every covering of the torus fiber of $N$ extends to a fiberwise covering of $N$. However the canonical $\mathbb{Z} / r \times \mathbb{Z} / r$ covering of the fiber always does extend; if we write $\pi_{1}(N)$ as $\mathbb{Z}^{2} \rtimes \mathbb{Z}$ then this cover corresponds to the subgroup $r \mathbb{Z}^{2} \rtimes \mathbb{Z}$. Note that this subgroup depends on the choice of splitting $\mathbb{Z} \rightarrow \pi_{1}(N)$ and is usually not a normal subgroup. Thus the resulting fiberwise covering is not unique and not usually a Galois covering. However, the resulting covering space is isomorphic to the original link, since the action of $\mathbb{Z}$ on $r \mathbb{Z}^{2}$ is the same as its action on $\mathbb{Z}^{2}$.

To confirm orientations in the above proof, consider the quotient-cusp with ma$\operatorname{trix} A=\left(\begin{array}{cc}a & 1 \\ a d-1 & d\end{array}\right)$. The resolution graph in this case is

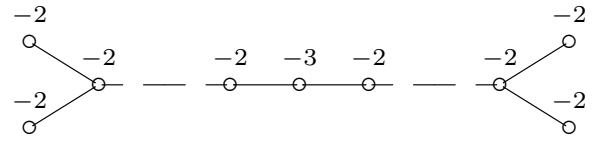

with respectively $a-1$ and $d-1$ vertices weighted -2 before and after the -3 on the central string. The universal abelian cover has degree 16 in this case and the above proof shows it can be built up by the sequence of covers corresponding to the decreasing chain of subgroups $\pi_{1}(N) \supset\langle V, z\rangle \supset\langle V, s\rangle \supset H=\left\langle V_{1}, s\right\rangle$. The first cover is the canonical two-fold cover by a cusp. The resolution graph for this cusp has strings of $2 a-3$ and $2 d-3$ weights -2 separated by -3 's. Next is the double cover of this cusp given by the subgroup $\langle V, s\rangle \subset\langle V, z\rangle$. This is the double cover in the circle direction, so it leads to a double cover of the resolution graph. The final cover is a $\mathbb{Z} / 2 \times \mathbb{Z} / 2$ fiberwise cover of the result, since $V / V_{1}=\mathbb{Z} / 2 \times \mathbb{Z} / 2$. This does not change the resolution graph. Thus, we have the right resolution graph.

\section{Other COVERS, COVERS OF CUSPS}

In this section we make the following miscellaneous observations.

\section{Proposition 4.1.}

1. The quotient-cusp classified by $\left(\begin{array}{ll}a & b \\ c & d\end{array}\right)$ has a natural $4 b$-fold abelian cover giving the same complete intersection cusp as the 16b-fold universal abelian cover. The latter is a $\mathbb{Z} / 2 \times \mathbb{Z} / 2$ fiberwise cover of the former.

2. Every cusp can be covered by a complete intersection cusp - even by a hypersurface cusp.

3. Not every cusp has an abelian cover by a complete intersection cusp.

It seems likely that there even exist cusps that have no Galois cover by a complete intersection, but we have not checked this.

Before we prove this proposition we need to recall the "discriminant group" of a singularity (for more details on the following, see e.g., [6]). 
Given any isolated complex surface singularity $(V, o)$, let $X$ be a tubular neighborhood of the exceptional divisor of a good resolution so $N=\partial X$ is the link of the singularity. Then $H_{2}(X) \cong \mathbb{Z}^{n} \subset H_{2}(X ; \mathbb{Q}) \cong \mathbb{Q}^{n}$ where $n$ is the number of exceptional curves in the resolution. Denote by $S$ the intersection form on these groups and define

$$
H_{n}(X)^{\#}=\left\{v \in H_{n}(X ; \mathbb{Q}): S(v, w) \in \mathbb{Z} \text { for all } w \in H_{2}(X)\right\} .
$$

The embedding $H_{2}(X) \rightarrow H_{2}(X)^{\#}$ can be identified with the map $H_{2}(X) \rightarrow$ $H_{2}(X, N)$, so the long exact sequence in homology identifies the discriminant group $D:=H_{2}(X)^{\#} / H_{2}(X)$ with the torsion of $H_{1}(N)$. The intersection form $S$ induces on $D$ a natural non-singular pairing

$$
D \otimes D \rightarrow \mathbb{Q} / \mathbb{Z}, \quad v \otimes w \mapsto S(v, w) \bmod \mathbb{Z} .
$$

This is the torsion linking pairing of $N$.

If $K \subset D$ is a subgroup then we get an induced non-singular pairing

$$
K \otimes\left(D / K^{\perp}\right) \rightarrow \mathbb{Q} / \mathbb{Z}
$$

where $K^{\perp}$ is orthogonal complement of $K$ under the pairing, so $D / K^{\perp}$ is canonically isomorphic to the dual $\hat{K}=\operatorname{Hom}(K, \mathbb{Q} / \mathbb{Z})$ and hence non-canonically isomorphic to $K$ itself.

If $N$ is a rational homology sphere then the universal abelian cover of $N$ is the Galois cover of $N$ determined by the natural homomorphism $\pi_{1}(N) \rightarrow H_{1}(N)=D$. Thus any subgroup $K \subset D$ determines an abelian cover of $N$ : the Galois cover with covering transformation group $D / K$. The annihilator $K^{\perp}$ of $K$ gives a cover that we call the dual cover for $K$; its covering transformation group is $D / K^{\perp}$. The dual cover for $D$ is thus the universal abelian cover.

For a cyclic subgroup generated by an element $[v] \in D$ the dual cover can be described in terms of its extension to a branched cover of the resolution $X$, branched along the exceptional divisor. If $v=\sum \frac{c_{i}}{d_{i}}\left[E_{i}\right]$, where $\left[E_{i}\right]$ are the homology classes of the exceptional curves $E_{i}$ of the resolution, then the branching along $E_{i}$ is of degree $d / d_{i}$, where $d$ is the order of the $[v]$ (hence the degree of the covering). The resulting branched cover of $X$ may have quotient singularities at intersection points of the $E_{i}$. After resolving these one obtains a (possibly non-minimal) resolution of the cyclic covering of the given singularity. This will be illustrated in the following.

Proof of Part 1 of Proposition 4.1. Using the notation just introduced, consider the following element $v \in D$ of order 2 for a quotient-cusp of the Introduction (coefficients of $v$ are in parentheses and omitted if zero - with vertices numbered $-1,0,1, \ldots, k+2$ in the obvious way, $\left.v=\frac{1}{2}\left(\left[E_{k+1}\right]+\left[E_{k+2}\right]\right)\right)$

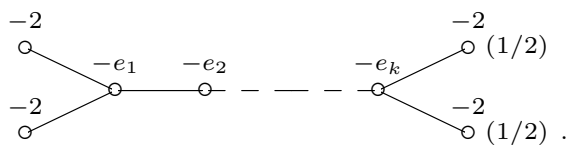

The double cover is

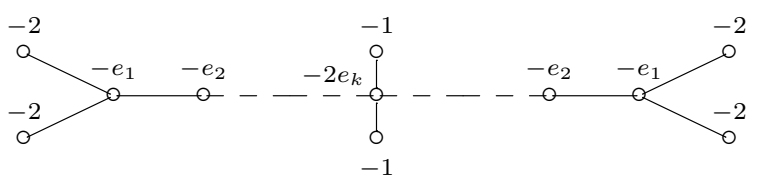


which blows down to

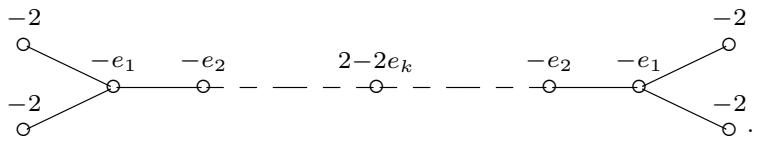

Similarly, the element $w=\frac{1}{2}\left(\left[E_{-1}\right]+\left[E_{0}\right]\right)$ gives a double cover by a quotientcusp, while the element $v+w$ gives the canonical double cover by the cusp of the Introduction. These elements $v$ and $w$ thus generate a Klein four-group $K \cong$ $\mathbb{Z} / 2 \times \mathbb{Z} / 2 \subset D$ whose dual covering is the canonical covering by a cusp followed by the canonical double covering that doubles the resolution cycle. Denote this 4 -fold covering of the links $N_{2} \rightarrow N$.

Now this $K$ is self-orthogonal with respect to the linking form, so $K \subset K^{\perp}$, so the dual covering $N_{3} \rightarrow N$ determined by $K^{\perp}$ factors through the covering just constructed. It is not hard to check that $N_{3} \rightarrow N_{2}$ is a $\mathbb{Z} / b$-fold cover, but we do not need this. The universal abelian cover $\tilde{N} \rightarrow N$ factors through $N_{3}$ and the covering transformation group for $\tilde{N} \rightarrow N_{3}$ is $K$. Since this is $\mathbb{Z} / 2 \times \mathbb{Z} / 2$ acting in the fibers of $\tilde{N}$, the quotient is isomorphic to $\tilde{N}$ again. This completes the proof of the first point of the above proposition.

If the link $N$ of an isolated complex surface singularity $(V, o)$ is not a rational homology sphere then there is no natural epimorphism $\pi_{1}(N) \rightarrow D$ to the discriminant group, hence no natural Galois cover with covering transformation group $D$. However, if $(V, o)$ is a cusp then the different epimorphisms of $H_{1}(N)=\mathbb{Z} \oplus D$ to $D$ are related by automorphisms of $\pi_{1}(N)$, and hence by automorphisms of $(V, o)$ (see [13]). So there is a natural cover up to automorphisms, called the discriminant cover. Hence for any subgroup $K \subset D$ we may still speak of the cover for $K$ and the dual cover for $K$, meaning the covers with covering transformation group $D / K$ respectively $D / K^{\perp}$.

The following proposition will be useful.

Proposition 4.2. If $D$ is the discriminant group for a cusp $(V, o)$ and $K \subset D a$ subgroup, then the cover and the dual cover of $(V, o)$ for $K$ are mutually dual cusps. In particular, taking $K=\{1\}$, then the discriminant cover of $(V, o)$ is the dual cusp of $(V, o)$.

Proof. The link $N$ of the cusp is a torus bundle with monodromy $A$ say. We can consider $N$ to be $T^{2} \times[0,1]$ with the two boundary components identified by $A$. Replacing $T^{2} \times\{0\} \subset N$ by a tubular neighborhood and excising the interior of this neighborhood, we see $H_{q}\left(N, T^{2}\right) \cong H_{q}\left(T^{2} \times[0,1], T^{2} \times\{0,1\}\right) \cong H_{q-1}\left(T^{2}\right)$. The long exact homology sequence for the pair $\left(N, T^{2}\right)$ thus leads to a long exact sequence

$$
\cdots \rightarrow H_{2}(N) \rightarrow H_{1}\left(T^{2}\right) \stackrel{A-I}{\longrightarrow} H_{1}\left(T^{2}\right) \rightarrow H_{1}(N) \rightarrow \operatorname{Ker}\left(H_{0}\left(T^{2}\right) \rightarrow H_{0}\left(T^{2}\right)\right)=\mathbb{Z} .
$$

This shows that the torsion $D$ of $H_{1}(N)$ is the image of $H_{1}\left(T^{2}\right) \rightarrow H_{1}(N)$ and is isomorphic to $\mathbb{Z}^{2} /(A-I) \mathbb{Z}^{2}$.

If $C$ is a 1 -cycle in $T^{2}$ then the 2 -chain $C \times[0,1] \subset N$ has boundary $(A-I) C$. It follows that the torsion linking form on $D=\mathbb{Z}^{2} /(A-I) \mathbb{Z}^{2}$ is given by $\ell([v],[w])=$ $\left((A-I)^{-1} v\right) . w(\bmod \mathbb{Z})$, where $v, w \in \mathbb{Z}^{2}$ and $v . w$ is the standard skew-symmetric form on $\mathbb{Z}^{2}$ (= intersection form on $H_{1}\left(T^{2}\right)$ ).

Suppose now that $K=W /(A-I) \mathbb{Z}^{2} \subset D$ and write $K^{\perp}=W^{\prime} /(A-I) \mathbb{Z}^{2}$. Then lemma 4.3 below implies $W^{\prime}=\frac{1}{\Delta}(A-I)^{-1} W$, where $\Delta=[D: K] /|\operatorname{det}(A-I)|$. 
The cover and the dual cover for $K$ of the given cusp have monodromy given by the action of $A$ on $W$ and $W^{\prime}$ respectively. Let $w_{1}, w_{2}$ be an oriented basis of $W$ and put $w_{1}^{\prime}=\frac{1}{\Delta}(A-I)^{-1} w_{1}, w_{2}^{\prime}=\frac{1}{\Delta}(A-I)^{-1} w_{2}$. Then $w_{1}^{\prime}, w_{2}^{\prime}$ is a reverse-oriented basis for $W^{\prime}$, since $(A-I)^{-1}$ has negative determinant (namely $\frac{1}{2-\operatorname{tr}(A)}$ ). But the matrix of $A$ with respect to $w_{1}, w_{2}$ equals the matrix of $A$ with respect to $w_{1}^{\prime}, w_{2}^{\prime}$ since $A$ and $(A-I)^{-1}$ commute. Thus the two cusps are mutual duals.

We used the following lemma, whose proof is an exercise.

Lemma 4.3. Let u.v denote the skew-symmetric form on $\mathbb{Q}^{2}$ induced by the standard non-singular skew-symmetric form on $\mathbb{Z}^{2}$. If $U \subset \mathbb{Q}^{2}$ is a $\mathbb{Z}$-lattice of covolume $\Delta$ (covolume is determinant of the matrix formed by an oriented basis) then the lattice

is $\frac{1}{\Delta} U$.

$$
\{v: v . u \in \mathbb{Z} \text { for all } u \in U\}
$$

Proof of Parts 2,3 of Proposition 4.1. Suppose we have a cusp given by the monodromy matrix $A=\left(\begin{array}{ll}a & b \\ c & d\end{array}\right)$. Assume $a \neq 0$ (if $a=0$ take $H=\mathbb{Z}^{2}$ in the following). Then $A$ takes the subspace $H$ of $\mathbb{Z}^{2}$ generated by $\left(\begin{array}{l}0 \\ 1\end{array}\right),\left(\begin{array}{l}a \\ c\end{array}\right)$ to itself by the ma$\operatorname{trix}\left(\begin{array}{cc}0 & -1 \\ 1 & t\end{array}\right)$, where $t=\operatorname{tr}(A)=a+d$. Thus the cover defined by the subgroup $H \rtimes \mathbb{Z} \subset \pi_{1}(N)$ is either the cusp with resolution graph consisting of a cycle with one vertex weighted $-t$ or the dual cusp of this, according as the above basis is oriented correctly or not, i.e., whether $a<0$ or $a>0$. In any case, by going to the discriminant cover if necessary (see Proposition 4.2), we get the cusp with resolution graph dual to the above length 1 cycle, so it is a cycle of $t-3-2$ 's and one -3 . This is a hypersurface cusp, proving the second statement of the proposition. Since this is a fiberwise cover, this hypersurface cusp not only covers, but is also covered by the original cusp. (In fact any two cusps with the same trace of their monodromy are mutual fiberwise covering spaces, see [1].)

For part 3 of the Proposition, a necessary condition that a cusp $(V, o)$ have no finite abelian cover by a complete intersection is that

- neither $(V, o)$ nor the dual cusp $\left(V^{*}, o\right)$ is a complete intersection.

We shall see that if

- the discriminant group $D$ has prime order $p$ say,

then this necessary condition is also sufficient. Indeed, suppose that $(V, o)$ and $\left(V^{*}, o\right)$ are not complete intersections. If $D$ has prime order then any abelian cover is either a cover in the circle direction or a cover in the circle direction of the discriminant cover. The resolution graph of the abelian cover is therefore a cyclic covering of the resolution graph or the dual resolution graph. By Proposition 2.5 this cannot be the resolution graph of a complete intersection.

An explicit example of this is the cusp with resolution cycle $[2,4,2,2,5]$ with discriminant group of order 61 . This cusp is self-dual and since the cycle is of length 5 it is not a complete intersection.

\section{The EXPlicit Universal ABELIAN COVER}

Let $(V, p)$ be the quotient-cusp classified by $\left(\begin{array}{ll}a & b \\ c & d\end{array}\right)$. We will write down explicit equations and an explicit group action for the universal abelian cover. 
Let $\omega$ be a primitive $4 b$-th root of 1 , and consider the diagonal $4 \times 4$ matrices

$$
\begin{aligned}
& S_{1}=\left[-\omega^{a}, \omega^{a}, \omega, \omega\right] \\
& S_{2}=\left[\omega^{a},-\omega^{a}, \omega, \omega\right] \\
& S_{3}=\left[\omega, \omega,-\omega^{d}, \omega^{d}\right] \\
& S_{4}=\left[\omega, \omega, \omega^{d},-\omega^{d}\right] .
\end{aligned}
$$

Let $G=\left\langle S_{1}, S_{2}, S_{3}, S_{4}\right\rangle$ be the abelian group they generate. We will show that $|G|=16 b$, its group structure depending on the parity of $c$.

Theorem 5.1. Consider the complete intersection cusp singularity $(X, o)$ given by the equations

$$
x^{2}+y^{2}=u^{\alpha} v^{\beta} ; \quad u^{2}+v^{2}=x^{\gamma} y^{\delta},
$$

where $\alpha, \beta, \gamma, \delta \geq 0$ satisfy

$$
\alpha+\beta=2 a ; \quad \gamma+\delta=2 d ; \quad \alpha \equiv \beta \equiv \gamma \equiv \delta \equiv c(\bmod 2) .
$$

Then

1. $G$ is an abelian group of order $16 b$ which acts freely on a punctured neighborhood of o in $X$,

2. $(X / G, o)$ is isomorphic to $(V, p)$

3. $(X, o) \rightarrow(V, p)$ is the universal abelian cover of $(V, p)$.

The proof shows that $G$ in fact acts freely on the smooth affine variety $X-\{o\}$ unless $\alpha \beta=\gamma \delta=0$. Note also that the equations for the cusp are not in the standard form of [3]; however, they are in the general form we use in [10], and should be considered as the analog of "Brieskorn complete intersections."

We describe $G$ first. Denote

$$
T=S_{1} S_{2}^{-1}=[-1,-1,1,1]
$$

Lemma 5.2. Every element of $G$ may be written uniquely as

$$
S_{1}^{j} T^{k} S_{3}^{l}, \quad 0 \leq j \leq 4 b-1,0 \leq k, l \leq 1 .
$$

Proof. First note that $G$ contains $-I$, equal to $S_{1}^{2 b}$ for $a$ odd, and $S_{1}^{2 b} T$ for $a$ even. Since

$$
S_{2}=T S_{1}, \quad S_{4}=-T S_{3}, \quad S_{3}^{2}=S_{1}^{2 d} T^{c},
$$

every element of $G$ may be written as desired. For uniqueness consider

$$
S_{1}^{j} T^{k} S_{3}^{l}=\left[(-1)^{j+k} \omega^{a j+l},(-1)^{k} \omega^{a j+l},(-1)^{l} \omega^{j+d}, \omega^{j+d l}\right],
$$

with $j, k, l$ as in the Lemma. The quotient of the last two entries shows $l$ is determined. The quotient of the second term by the $a$-th power of the last then shows $k$ is determined. This implies the uniqueness.

Lemma 5.3. 1. For c even, $G \cong C_{4 b} \times C_{2} \times C_{2}$, with factors generated by $S_{1}, T$, and $S_{1}^{-d} T^{c / 2} S_{3}=[-1,1,-1,1]$.

The only non-identity elements of $G$ with $\geq 2$ eigenvalues equal to 1 are the 6 elements with $2+1$ 's and $2-1$ 's.

2. For $c$ odd, $G \cong C_{4 b} \times C_{4}$, with factors generated by $S_{1}$ and $S_{1}^{d} S_{3}^{-1}=$ $\left[(-1)^{d} i^{c}, i^{c},-1,1\right]$.

The only non-identity elements of $G$ with $\geq 2$ eigenvalues equal to 1 are $\pm T$. 
Proof. The proof is straightforward and left to the reader. Note that in the second case $\left(S_{1}^{d} S_{3}^{-1}\right)^{2}=T$.

Lemma 5.4. $G$ acts on $X$, freely off finitely many points (only 0 , unless $c$ is even and $\alpha \beta=\gamma \delta=0)$.

Proof. $G$ acts on $X$ because the generators $S_{i}$ each transform each defining equation by a character. The origin is the only point of $X$ with at least 3 coordinates equal to 0 . A non-trivial element of $G$ with $\geq 2$ eigenvalues equal to 1 must have fixed locus a coordinate plane given by setting two coordinates equal to 0 . Such a plane intersects $X$ in only finitely many points.

The following facts are straightforward. $X$ has one singular point, at 0 . The blowing-up $X_{1}$ of $X$ at 0 is normal. The exceptional fiber $E$ (= projectivized tangent cone of $(X, o))$ consists of either $4 \mathbb{P}^{1}$ 's $(a, d>1)$ :

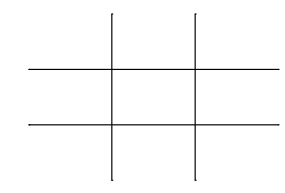

or $2 \mathbb{P}^{1}$ 's $(a=1$ or $d=1)$ :

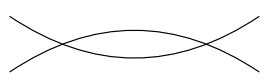

In the first case, $X_{1}$ has singularities of type $A_{2 a-3}$ and $A_{2 d-3}$ at alternate intersection points of the irreducible exceptional curves. In the second case, $X_{1}$ has two $A_{2 d-3}$ singularities. In particular, $(X, o)$ is the cusp singularity of Theorem 1.3 .

Since $G$ acts freely on the cusp $(X, o)$ off the origin, the quotient $(X / G, o)$ must be either a cusp or a quotient-cusp. But the second (non-Gorenstein) case must occur; if

$$
\eta=d x \wedge d y /\left(f_{u} g_{v}-f_{v} g_{u}\right)
$$

is the standard nowhere-zero holomorphic 2-form on $X-\{o\}(f, g$ the defining equations), one finds

$$
S_{1}^{*} \eta=-\eta
$$

So, $(X / G, o)$ is a quotient-cusp. If we show $(X / G, o)$ has type $\left(\begin{array}{ll}a & b \\ c & d\end{array}\right)$ then we are done, since the abelian cover $(X, o) \rightarrow(X / G, o)$ has degree $16 b$, which is the degree of the universal abelian cover by earlier computations.

We shall satisfy ourselves by carrying out this calculation for $b=1$. This is not as special as it may seem; we show later that it implies the general case that $b$ is odd.

So, let us assume $b=1$, and consider $a, d>1$ (the case that $a$ or $d$ is 1 is similar). We will examine the quotient $X_{1} \rightarrow X_{1} / G$. $G$ acts on the graph of $E$ as $C_{2} \times C_{2}$, either rotating by $180^{\circ}$ or reflecting in a diagonal. So, the quotient of $E$ by $G$ is one irreducible curve with 2 distinguished points. Further, off the 4 singular points of $E$ the stabilizer of any point is $\pm I$ (which acts trivially on $E$ ). This is because $\pm I$ are the only scalar matrices on $G$. We conclude that on $X_{1} / G$, the image of $E$ is a rational curve with two locally irreducible singular points; and $X_{1} / G$ is smooth off 
these two points. Further, the map $X_{1} \rightarrow X_{1} / G$ at a general point of $E$ consists of a degree 2 cover branched along $E$, followed by an 8 -to- 1 covering map.

To find the singular points of $X_{1} / G$, choose a singular point of $E$. Its stabilizer $H$ has order 8. We do a local calculation. Assume, e.g., $c$ is even. Then it is straightforward to find local analytic coordinates so that $X_{1}$ is defined by

$$
x_{1}^{2}+y_{1}^{2}=u^{2 a-2},
$$

$E$ is defined by $u=0$, and $H$ acts via $\left[-i^{a-1}, i^{a-1}, i\right]$ and $[-1,-1,1]$. Writing down explicit invariants, one computes that the quotient is a $D_{a+1}$-singularity; further, the image of the curve $u=x_{1} \pm y_{1}=0$ will intersect the resolved singularity on the long end. Therefore, the dual graph of the minimal resolution $\tilde{X}$ of $X_{1} / G$ is

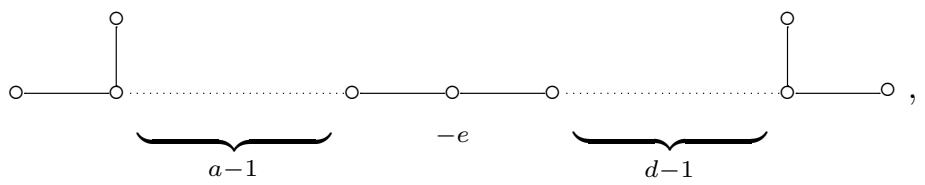

where all but the central curves are -2 -curves.

It remains only to show that $e=3$, as a calculation then shows that the above is the $\left(\begin{array}{cc}a & 1 \\ a d-1 & d\end{array}\right)$ cusp quotient. This is achieved by computing the divisor on $\tilde{X}$ of the function $g=x^{4}-u^{4}$ (this function on $X$ is easily checked to be $G$-invariant). On $X_{1}$, the divisor of $g$ consists of $E$ counted with multiplicity 4, plus 16 smooth curves, 4 per component of $E$, intersection $E$ transversally at 4 distinct non-singular points. By the branching behavior of $X_{1} \rightarrow X_{1} / G$ along $E$, we conclude that $g$ vanishes twice on $\tilde{X}$ along the central curve, and the proper transform of $\{g=0\}$ consists of two smooth curves intersecting the central curve transversally at distinct points. A standard calculation then shows $e=3$.

We promised to show that the general case of $b$ odd follows from the just completed case $b=1$ (the same argument will show that for general $b$ it suffices prove the cases that $b$ is a power of 2 ).

We first note that it suffices to show that $(X, o) \rightarrow(X / G, o)$ is a universal abelian cover. For if $(X / G, o)$ has type $\left(\begin{array}{ll}a_{1} & b_{1} \\ c_{1} & d_{1}\end{array}\right)$, then the discriminant of $(X / G, o)$ would be

$$
16 b_{1}=|G|=16 b
$$

so $b=b_{1}$. Also, by Theorem 1.3, $\left\{a_{1}, d_{1}\right\}$ is determined by the cusp cover, so, up to order, $a=a_{1}, d=d_{1}$. Since $a_{1} d_{1}-b_{1} c_{1}=1$, we conclude that $(X / G, o)$ is of type $\left(\begin{array}{ll}a & b \\ c & d\end{array}\right)$, as desired.

Now let $G_{1} \subset G$ be the subgroup of order 16 generated by $S=S_{1}^{b}$ and the other 1 or 2 generators described in Lemma 5.3. This is exactly the $G$ one would get if $b=1$ and, under the assumption that $b$ is odd, one easily checks that it is the correct action. We have already shown that $(X, o) \rightarrow\left(X / G_{1}, o\right)$ is a universal abelian cover; this implies that the larger abelian quotient $(X, o) \rightarrow(X / G, o)$ is also a universal abelian cover.

\section{REFERENCES}

[1] F. Hirzebruch, Hilbert modular surfaces. Enseignement Math. (2) 19 (1973), 183-281.

[2] U. Karras, Eigenschaften der lokalen Ringe in zweidimensionalen Spitzen. Math. Ann. 215 (1975), 117-129. 
[3] U. Karras, Deformations of cusp singularities. Several complex variables Proc. Sympos. Pure Math., Vol. XXX, Part 1, Williams Coll., Williamstown, Mass., 1975, (Amer. Math. Soc., Providence, R.I., 1977), 37-44.

[4] Y. Kawamata, Crepant blowing-up of 3-dimensional canonical singularities and its application to degeneration of surfaces, Ann. of Math. 127 (1988), 93-163.

[5] H. B. Laufer, Taut two-dimensional singularities. Math. Ann. 205 (1973), 131-164.

[6] E. Looijenga, J. Wahl, Quadratic functions and smoothing surface singularities. Topology 25 (1986), 261-291.

[7] W.D. Neumann, A calculus for plumbing applied to the topology of complex surface singularities and degenerating complex curves, Trans. Amer. Math. Soc. 268 (1981), 299-343.

[8] - Abelian covers of quasihomogeneous surface singularities, Singularities, Arcata 1981, Proc. Symp. Pure Math. 40 (Amer. Math. Soc. 1983), 233-243.

[9] W.D. Neumann, J. Wahl, Casson invariant of links of singularities, Comment. Math. Helv. 65 (1990), 58-78.

[10] Complete intersection singularities with homology sphere links (in preparation).

[11] H.C. Pinkham, Automorphisms of cusps and Inoue-Hirzebruch surfaces. Compositio Math. 52 (1984), 299-313.

[12] C.T.C. Wall, Real forms of cusp singularities. Math. Proc. Cambridge Philos. Soc. 99 (1986), 213-232.

[13] C.T.C. Wall, Real forms of cusp singularities. II. Math. Proc. Cambridge Philos. Soc. 102 (1987), 193-201.

Department of Mathematics, Barnard College, Columbia University, New York, Ny 10027

E-mail address: neumann@math.columbia.edu

Department of Mathematics, The University of North Carolina, Chapel Hill, NC $27599-3250$

E-mail address: jw@math.unc.edu 